\title{
Insight into indentation-induced plastic flow in austenitic stainless steel
}

\author{
Wenzhen Xia ${ }^{1, \star}$, Gerhard Dehm ${ }^{1}$, and Steffen Brinckmann ${ }^{1,2, \star}$ \\ ${ }^{1}$ Department of Structure and Nano-/Micromechanics of Materials, Max-Planck-Institut für Eisenforschung GmbH, 40237 Düssel- \\ dorf, Germany \\ ${ }^{2}$ Microstructure and Properties of Materials (IEK-2), Forschungszentrum Jülich, 52425 Jülich, Germany
}

Received: 11 February 2020

Accepted: 2 April 2020

Published online:

15 April 2020

(C) The Author(s) 2020

\begin{abstract}
The indentation-induced plasticity and roughness have been investigated intensively by experiments and simulations during the last decades. However, the precise mechanisms of how dislocation flow leads to pile-up formation are still not completely understood, although this is one of the initial steps causing surface roughening in tribological contacts at low loads. In this work, $\{001\}-$, $\{101\}$ - and $\{111\}$-grain orientations in an austenite stainless steel [(face-centered cubic (FCC) phase]) are indented with varying load forces. By using scanning electron-based methods and slip plane analysis, we reveal: (1) how slip-steps show the change of pile-up formation, (2) how the slip-plane inclination determines the dislocation flow and (3) how slip-plane interactions result in the final pile-up shape during indentation. We find that the flow direction transforms from the forward flow to the sideway at a transition angle of $55^{\circ}-58^{\circ}$ between the slip-plane and the surface. We use large displacement finite element method simulations to validate an inversion of the resolved shear stress at this transition angle. We provide insights into the evolution of plasticity in dislocation-mediated FCC metal indentations, with the potential application of this information for indentation simulations and for understanding the initial stage of scratching during tribology in the future.
\end{abstract}

\section{Introduction}

Nanoindentation has become a mature technique for mechanical property identification and small lengthscale materials science research [1-13]. The load versus penetration depth curve ( $\mathrm{P}-\mathrm{h}$ curve) is used to determine the elastic modulus and hardness via the
Oliver-Pharr method $[4,5,14,15]$. Indentation pop-in load is the force which the indentation transitions by a depth jump from the elastic to the elastoplastic regime. The investigation of the pop-in load reveals the theoretical strength and the contribution of initial dislocations [1]. The Nix-Gao model quantifies the indentation size effect (ISE), i.e., a decreasing hardness with increasing indentation depth, due to

Address correspondence to E-mail: w.xia@mpie.de; brinckmann@mpie.de 
geometrically necessary dislocations (GND) accumulation under the indenter $[6,16]$.

In addition to the ISE, dislocation motion results in the formation of a pile-up and surface steps. As the indenter penetrates the sample, the material moves downward and experiences a strain gradient, which results in dislocation motion toward the surface, which depends on the resolved shear stress in the slip direction due to the particular grain orientation. Once dislocations escape from the grains, slip-steps are left behind on the surface. The investigation of slip-steps tracks the plastic flow during nanoindentation. Tromas et al. [17] reconstructed 3D dislocation loops after the first indentation pop-in using nanoetching and atomic force microscopy (AFM) in $\mathrm{MgO}$. The experiments revealed that dislocations are nucleated in the area of maximum resolved shear stress (RSS) underneath the tip with a distance and travel toward the free surface on positively inclined slip-planes, as shown schematically in Fig. 1. The dislocations originating from underneath the indenter, however, did not fully account for the slip-step formation around the imprint, as stated by Velednitskaya et al. [18]. Tromas et al. [19] observed also slip-steps due to dislocations on outer planes, i.e., negatively inclined slip-planes. It should be noted that the nomenclature of positively and negatively inclined slip-planes was proposed by Nibur et al. [20, 21], who studied the influence of grain orientation and tip geometry on slip-step formation in face-centered cubic (FCC) metals. A Positively inclined slip-plane is a plane on which the dislocations move from the highest resolved shear stresses (i.e., underneath the tip) to the surface and away from the tip. The dislocations travel on the negatively inclined slip-planes from the outside bulk material to the surface and closer to the tip during indentation (see Fig. 1).

Researchers have also investigated the dislocation motion-induced pile-up and sink-in formation, which affect significantly the true contact area andhence - the identified mechanical properties [22-25]. Taljat and Pharr [25] verified that the sink-in is linked to the elastic Hertzian contact and that the pile-up evolves during the plastic regime. Eidel [26] disclosed using crystal plasticity finite element simulations (CP-FEM) that pile-ups form as a result of plastic flow on positively inclined $\{111\}\langle 110\rangle$ slip systems in FCC alloys. The author also observed that slip was dependent on the crystal orientation. Casals et al. [27] revealed by CP-FEM that the slip-system orientation governs the GND evolution and the sinkin/pile-up evolution. Wang et al. [28] used a conical indenter to penetrate the $\{001\}-,\{011\}$ - and $\{111\}$ surfaces of copper crystals. Their numerical results showed that the surface roughness is formed by pileups for this material (rather than sink-ins) and that two-, four- and six-fold symmetry exists on the $\{011\}$, $\{001\}$ and $\{111\}$ surfaces, respectively. However, CPFEM is a continuum model that does not account for the motion of individual dislocations and the slipstep evolution. The pile-up evolution could also be studied by molecular dynamics (MD) or dislocation

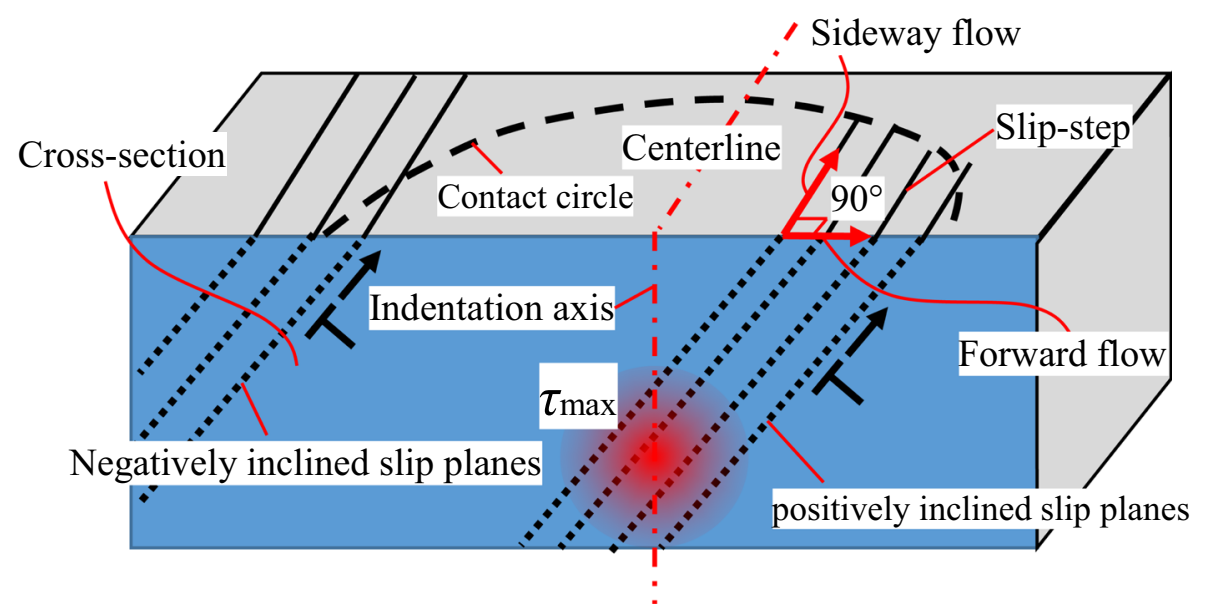

Figure 1 Schematic residual imprint after indentation and slipplanes with positive and negative inclination: $\tau_{\max }$ denotes the area of maximum resolved shear stress; the indentation axis is given by a dashed dotted line and the contact area by the dashed line.
Negatively inclined and positively inclined planes are separated by the centerline; the forward flow is in the radial direction, while the sideway flow is in the tangential direction. 
dynamics (DD). However, both methods are not ideal for this research question. MD simulates small domains and high velocities that might influence the pile-up formation. DD generally does not account for the influence of the pile-up onto the stress state. (No large deformation framework is used.) Hence, in the current progression, both methods are not able to advance the understanding of pile-up evolution.

The plasticity underneath the indented surface has also been studied experimentally and numerically. Zaafarani et al. [29] investigated the nanoindentationinduced lattice rotation in a (111) $\mathrm{Cu}$ single crystal through 3D electron backscatter diffraction (EBSD). The inner zone at the indenter axis is rotated by smaller angles, as compared to the outer zone that additionally has the opposite rotation direction to the inner zone. Demir et al. [30] used 3D-EBSD to measure GND densities beneath the imprints: GNDs distribute heterogeneously, and the GND density increases with increasing indentation depth. Robertson and Fivel [31, 32] and Gagel, et al. [33] used 3D DD simulations to mimic dislocation mechanisms during nanoindentation in a FCC crystal. Those authors neglected the large deformations that arise during pile-up formation and that change the stress state. Chang et al. [34] employed MD simulations and found that prismatic dislocation motion dominates the plasticity during nanoindentation in FCC metals. This prismatic slip leads to slip-steps that encircle the indentation axis. Begau et al. [35, 36] and Stukowski and Arsenlis [37] also observed the prismatic dislocation loops in their MD simulations. The MD simulations of Varillas et al. [38] studied the dislocation interaction, including prismatic loop formation, and identified the formation mechanisms of slip-step and pile-ups in FCC and BCC metals. However, these prismatic loops are not observed by conventional experiments, which have a slow indentation velocity $[9,32,39-41]$.

The previous extensive experiments and simulations have been shown that (1) pile-ups form during nanoindentation, (2) pile-up formation is linked to plasticity / dislocation motion, and (3) the crystal orientation determines the pile-up shape. However, the discrepancies between simulations and experiments [28, 31-37] (the inability of CP-FEM to predict slip-steps, the lack of accounting for the pile-up evolution in 3D DD, the very fast deformation rates in MD and the prediction of prismatic dislocation loops in the latter two simulation methods while experiments at conventional deformation rates show an abundance of conventional, i.e., non-prismatic, dislocations.) reveal that the precise link of dislocation flow, crystal orientation, pile-up formation and stress-state has not been thoroughly understood.

Toward this goal, this study on austenitic steel will investigate (1) how the evolution of slip-step patterns leads to the development of pile-ups, (2) how the slip-plane tilt angle determines dislocation flow and (3) how the interaction of slip-plane results in the specific pile-up shape. We compare the experimentally identified dislocation flow mechanisms with the results from finite element method (FEM) simulations and discuss how dislocations on specific glide planes lead to the specific pile-up. The motivation of this study is twofold: (1) To thoroughly understand the deformation that arises during nanoindentation, which has been used and continues to be used at the nanoscale to evaluate the mechanical properties of single phases. The use of an experimental technique is contingent on the complete understanding of the deformation mechanisms and (2) to provide a better understanding of the deformation that occurs during the transition from nanoindentation to nanoscratch tribology in the future, in which hard phases get in contact with ductile counter surfaces and result in local abrasion. This deformation mechanism is akin to nanoindentation and nanoscratch by a nanoindenter tip.

\section{Experimental procedure}

We used an austenitic stainless steel Fe-25Cr-20Ni with a stacking fault energy (SFE) of around $45 \mathrm{~mJ} /$ $\mathrm{m}^{2}$ [42]. To achieve coarse-grained microstructure, the as-received specimen was annealed at $1200{ }^{\circ} \mathrm{C}$ for $168 \mathrm{~h}$ and then water quenched. The large grains allow testing repeatedly in the same grain without grain boundary interference. The sample was cut by electrical discharge machining to avoid the formation of a thick deformation layer. Afterward, the surface was mechanically ground, polished by 3 and $1 \mu \mathrm{m}$ diamond suspensions as well as $50 \mathrm{~nm}$ colloidal silica OPS suspension. The polished specimens were etched using a V2A solution $\left(10 \mathrm{ml} \mathrm{HNO}_{3}, 0.30 \mathrm{ml}\right.$ Vogels pickling inhibitor, $100 \mathrm{ml} \mathrm{HCl}$ and $100 \mathrm{ml}$ $\mathrm{H}_{2} \mathrm{O}$ ) for $30 \mathrm{~s}$ to remove the minor deformation layer that was induced by the mechanical preparation. The following OPS polishing removed the chemical 
reaction layer. The etching and polishing steps were repeated for a number of iterations. The final step was a 2 min ethanol polishing to remove the retained OPS nanoparticles. After surface preparation, the grain orientations were inspected in a JEOL 6490 scanning electron microscope (SEM) with an EBSD detector. Three grains whose orientations are close to the $\{111\},\{101\}$ and $\{001\}$ planes were selected for nanoindentation to investigate the influence of grain orientation on plastic flow. The precise orientations of the grains were $\{182019\},\left\{2_{13}^{-} 13\right\}$ and $\left\{5_{6}^{-} 25\right\}$, respectively.

We used an Agilent/Keysight/KLA G200 nanoindenter in load-controlled mode with a spherical diamond tip. The G200 has ultrahigh resolutions of load and displacement (load: $50 \mathrm{nN}$, displacement: $\sim 0.01 \mathrm{~nm})$ and was calibrated before the experiment: load-frame stiffness, area-function and support spring stiffness. The measurements were started after the thermal drift rate was lower than $0.5 \mathrm{~nm} / \mathrm{s}$. We use a spherical indentation tip in order to analyze the crystal anisotropy and crystal plasticity. By using an axisymmetric tip, we eliminate the artifacts that arise due to corners of the indenter tip, e.g., Vickers tip. The roughness of the sample surface and the tip radius are $4 \mathrm{~nm}$ and $11.2 \mu \mathrm{m}$, respectively, as examined by a Keyence VK-X series 3D laser scanning confocal microscope. The maximum loads were 2, 5, $10,20,40$ and $80 \mathrm{mN}$ during nanoindentation on the three grains. Loading-unloading rates of $0.05,0.2$ and $0.5 \mathrm{mN} / \mathrm{s}$ were used. Since post-deformation electron microscopy inspection of indentations made at these rates did not reveal any difference in the imprint patterns (tests repeated for two different grain orientations), see supplementary material, we focus on the results of the $0.5 \mathrm{mN} / \mathrm{s}$ experiments and do not address the results of the other loading-unloading rates. After indentation, the surfaces were examined in a ZEISS Merlin field emission SEM with a secondary electron (SE) and a backscatter electron detector (BSD). An acceleration voltage of $3 \mathrm{kV}$, a probe current of $6 \mathrm{nA}$ and a working distance of $4.8 \mathrm{~mm}$ were applied to capture the SE images. The electron channeling contrast images (ECCI) were obtained at a voltage of $30 \mathrm{kV}$, a current of $2 \mathrm{nA}$ and a working distance of $6.8 \mathrm{~mm}$. We used an AFM (Veeco Digital Instruments, Plainview, New York, DI3100S-1) and a confocal microscopy ( $\mu$ surf,
NanoFocus Messtechnik GmbH, Germany) to inspect the indentation topographies.

\section{Results}

In this study, we focus on the plastic material response of the material and neglect the elastic response, since we observed in a previous study [41] that the load-displacement curve follows the Hertz equation and since the elastic deformation does not result in a surface topography that could be inspected by SEM.

\section{Pile-up formation depending on the slip- plane inclination angle}

SEM and confocal images of the $\{111\},\{101\}$ and $\{001\}$ grains after indention with $80 \mathrm{mN}$ are shown in Fig. 2. In order to present how the slip-steps evolve with increasing load, the steps after applying $2 \mathrm{mN}$ are sketched in Fig. $2 \mathrm{~g}-\mathrm{i}$ for the respective grains. (The SEM images after indention with $2 \mathrm{mN}$ and one exemplary $\mathrm{P}-\mathrm{h}$ curve are shown in supplemental material.) Please note that the dislocations travel on the positively inclined slip-planes during the initial elastoplastic stage of nanoindentation and the initial pile-up sites are determined by slip on the positively inclined planes. The pile-up formation contributes to the slip on the negatively inclined slip-plane [41].

The plastic flow into surface pile-ups is the results of dislocation motion on the slip-planes during indentation. This plastic flow is analyzed by SEM and confocal microscopy data from slip-steps and pileups that are produced by varying indentation forces. We project the plastic slip-plane bound flow onto the surface and identify two surface components: sideway along the slip-step and forward flow perpendicular to the slip-step (see Fig. 1). Namely, sideway flow is the component of the vector sum of all dislocations; the component is parallel to the slip-steps. Forward flow is the dislocation sum vector component that is in the surface plane and perpendicular to the slip-step. This identification of the surface component of the plastic flow allows to unravel the importance of the slip-plane inclination.

In the $\{111\}$ grain, three pile-up areas form as marked with blue circles, see Fig. 2d. When inspecting the slip-steps and pile-ups after applying a force of $2 \mathrm{mN}$, the dislocation motion is mostly sideways 
$\{111\}$

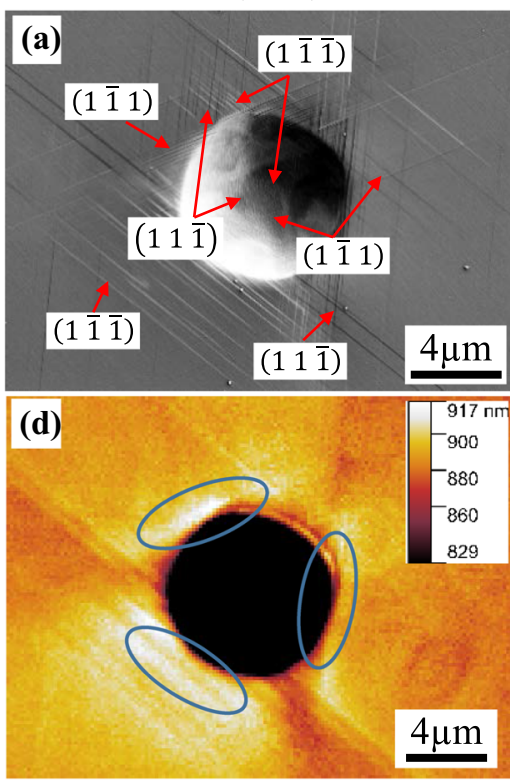

(g)
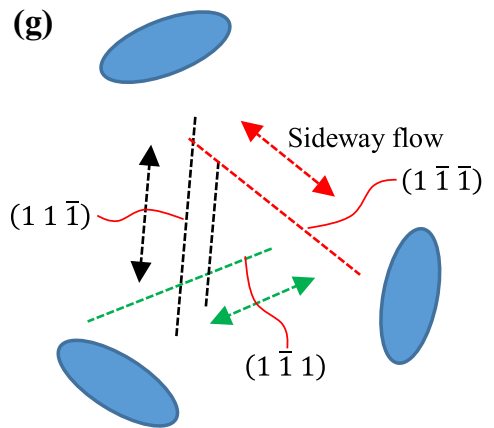

$\{101\}$
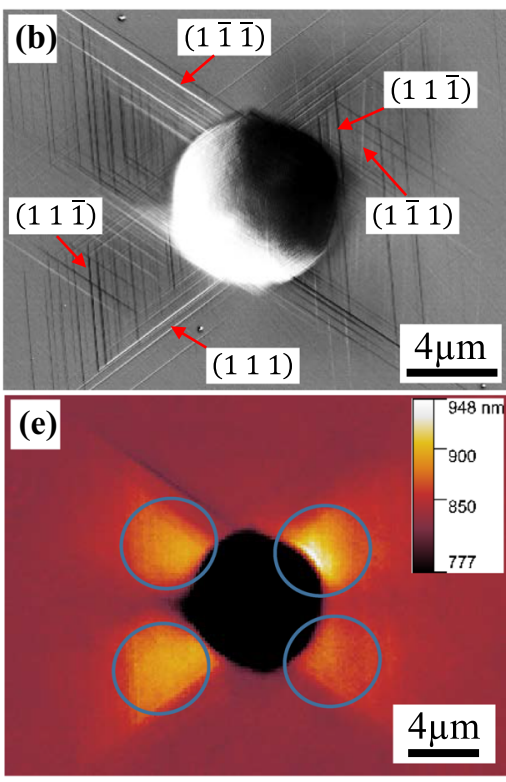

(h)

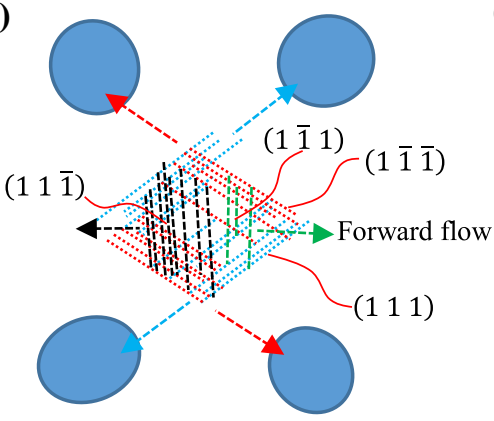

$\{001\}$

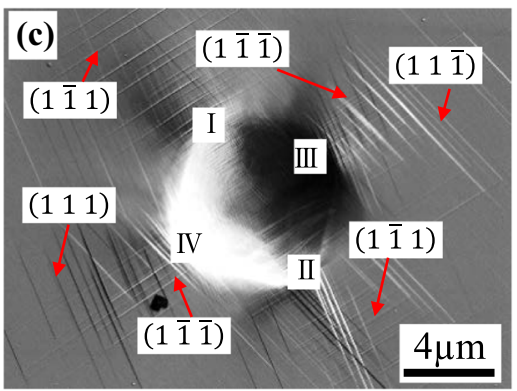

SEM

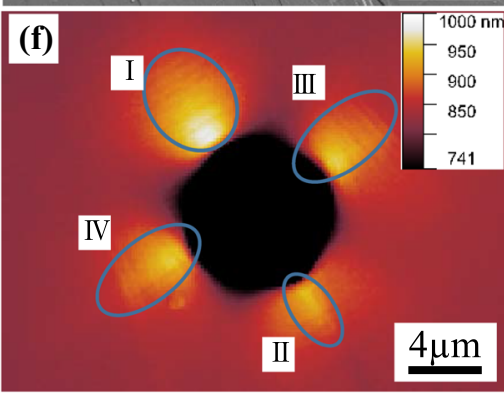

Confocal

image

(i)

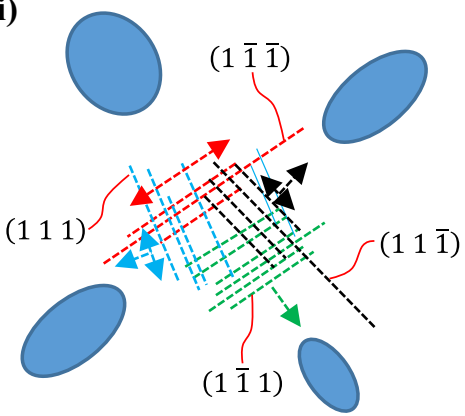

Sketch
Figure 2 Secondary electron SEM and confocal images of the 80$\mathrm{mN}$ imprints and sketches of the slip-steps after applying $2 \mathrm{mN}$.

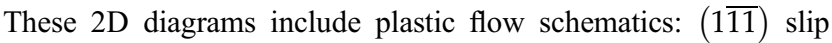
activity is shown in red; (1111) activity in green; (111) activity in

on the three positively inclined slip-planes. These slip-planes have an inclination angle $\alpha$ with the surface of $\sim 70^{\circ}$. In the $\{101\}$ grain, the slip-steps of $\left(1_{11}^{--}\right)$and (111) planes reveal that plasticity flows mostly to one side on these planes, as marked with red and blue arrows in Fig. 2h. Most dislocation plasticity occurs on these planes that are almost perpendicular to the surface (Fig. 2b) as evident from the height measurement in Fig. 2e. The dominant plastic flow on the $\left(11_{1}^{-}\right)$and $\left(1_{1}^{-} 1\right)$ planes is forward and away from the indenter, based on the slipstep evolution, as illustrated in Fig. $2 \mathrm{~h}$. The inclination angles of the $\left(11_{1}^{-}\right)$and $\left(1_{1}^{-} 1\right)$ planes are $\sim 35^{\circ}$. In the $\{001\}$ grain, the plastic flow on the positively black; and (111) activity in blue. Note that the arrows show the projection of the slip on the glide plane onto the surface plane [the supplemental information contains the corresponding SEM images of (g)-(i)].

inclined $\left(1_{1}^{-} 1\right)$ slip-plane $\left(\alpha=37^{\circ}\right)$ is mostly forward in the bottom right corner (II), as shown in Fig. 2f. In the upper left corner (I), the $\left(1_{1}^{-} 1\right)$ slip-planes are negatively inclined and secondary slip-systems that do not contribute to the initial pile-up, as discussed previously. On the $\left(11_{1}^{-}\right)$and (111) slip-planes $\left(\alpha=55^{\circ}\right.$ and $\alpha=58^{\circ}$, respectively), plasticity flows forward into areas (III) and (IV). Additionally, sideway plastic flow is observed into zones (I) and (II) on these two planes. The sideway slip on the $\left(1_{1}^{-\overline{1}}\right)$ plane $\left(\alpha=73^{\circ}\right)$ occurs into areas (III) and (IV), as shown in Fig. $2 \mathrm{i}$. It should be noted that the $\left(1_{1}^{-\overline{1}}\right)$ 
slip-steps are only observed in areas (III) and (IV), but not in areas (I) and (II).

From these results, we categorize the plastic flow (forward / sideway) for each slip-plane depending on the inclination angle of that plane to the surface. The results are shown in Fig. 3a, which reveal that there is a transition angle of $\alpha_{\text {Trans }}=55^{\circ}-58^{\circ}$. When the inclination angle is smaller than that value, the plasticity flows forward on the slip-planes; if the inclination angle is higher, the plasticity will flow sideways on the slip-planes. Figure $3 b$ sketches the plastic flow direction as a function of slip-plane inclination angle, based on the finding obtained from Fig. 3a. The direction of plastic flow is shown with arrows, whose length denotes the magnitude of flow in the two principal directions: forward flow and sideway flow.

\section{Interaction between slip-planes}

In the previous section, the dependence of the plastic flow on the slip-plane inclination angle was discussed. Additionally, the pile-up size and shape are governed by the slip-plane interaction. We study one interaction pair to show the analysis method and the material scientific findings.

Figure 4 shows the bottom left pile-up in the $\{101\}$ grain and the intersecting $\left(11_{1}^{-}\right)$and (111) slip-planes. After indentation with $10 \mathrm{mN}$, the first slip-step ' $\mathrm{a}$ ' on a (111) plane, which occurs outside the parallelogram, is located at a distance of $\sim 700 \mathrm{~nm}$ to the indentation centerline (the indentation centerline of a particular normal direction runs through the center of the indentation imprint and is parallel to the slipsteps of this plane. This centerline separates the positively and negatively inclined slip-planes), as shown in Fig. 4a. Please note that this distance remains constant during indentation, i.e., the distance from the maximum elevation (marked with ' $b$ ' in Fig. $4 \mathrm{~b}$ and a blue arrow in Fig. $4 \mathrm{c}$ ) to the (111) centerline is the same after indentation with $80 \mathrm{mN}$. Hence, while the imprint increases in size during indentation, the distance of the maximum elevation to the centerline remains constant. Moreover, note that the slip-steps on the bottom (111) slip-planes are longer than on the parallel upper planes.

The dislocation characterization is shown in Fig. 5 for the $\{101\}$ grain after applying $80 \mathrm{mN}$, using the ECCI technique with controlled two beam conditions. The sample is tilted and rotated to obtain four different two-beam conditions away from the zone axis (see Fig. 5a-d). Using these diffraction conditions, we determine the dislocations' Burgers vectors using the $\mathbf{g} \cdot \mathbf{b}=0$ invisibility criterion [43]. Please note that most dislocations are on the $\left(11_{1}^{-}\right)$slip-planes and three possible Burgers vectors exist (see Table 1). The dislocations in the red ellipse are visible for $\mathbf{g}=(022)$, (111), $\left(\frac{-}{2} 00\right)$ while invisible for $\mathbf{g}=\left({ }_{1}^{-} 11\right)$. Therefore, these dislocations have a Burgers vector of $\left[{ }_{1}^{-} 0_{1}^{-}\right]\left(11_{1}^{-}\right)$. In the green ellipse, the dislocations are weakest for $\mathbf{g}=\left(\frac{-}{2} 00\right)$, compared to the other three gvectors. Hence, the Burgers vectors of these dislocations are $\left[0_{11}^{--}\right]\left(11_{1}^{-}\right)$.

\section{Discussion}

The pile-up patterns depend on the grain orientation: Multiple triangles in the $\{111\}$ grain, butterfly shapes in the $\{101\}$ grain and the cross shape in $\{001\}$ grain were also observed during previous studies [21, 26, 28, 44-47]. Wang et al. [28] reported that the pile-up pattern on a $\{111\}$ surface has a sixfold
Figure 3 a Categorization of the flow direction depending on the inclination angle $\alpha$ of slip-plane with the free surface and $\mathbf{b}$ schematic diagram of metal flow on the slip-planes. The length of the arrows denotes the amount of flow in that direction (forward flow or sideway flow).
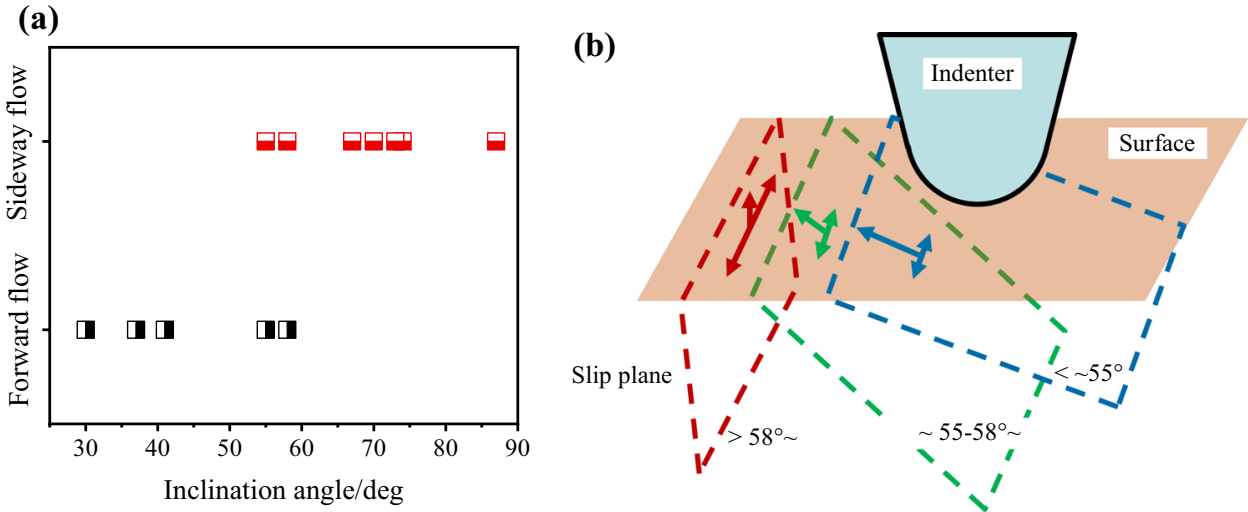

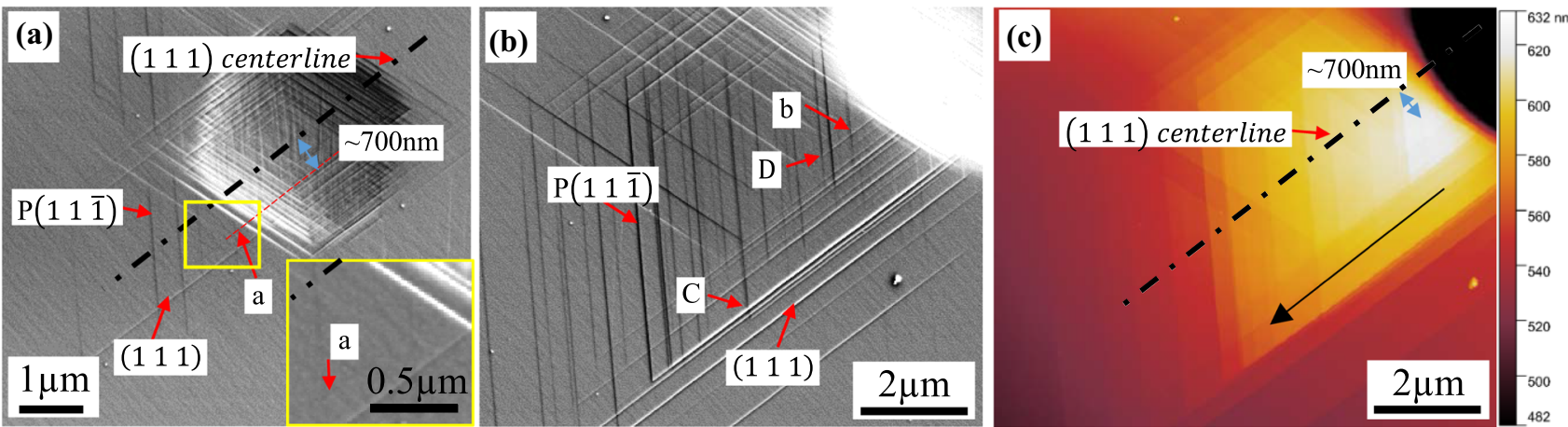

Figure 4 Dislocation interaction on intersecting (11) and (111) planes. Indentation forces of $\mathbf{a} 10 \mathrm{mN}$ and $\mathbf{b}, \mathbf{c} 80 \mathrm{mN}$ were applied in the $\{101\}$ grain: $\mathbf{a}, \mathbf{b}$ SEM images and $\mathbf{c}$ confocal height measurement.

Figure 5 Characterization of dislocations in the $\{101\}$ grain using ECCI technique with controlled two beam conditions: $\mathbf{a} \mathbf{g}_{1}=(022) ; \mathbf{b}$ $\mathbf{g}_{2}=(111) ; \mathbf{c} \mathbf{g}_{3}=(\overline{1} 11) ; \mathbf{d}$ $\mathbf{g}_{4}=(\overline{2} 00)$. The dislocations are labeled with a red/green ellipse, depending on in which condition they disappear or become weak.
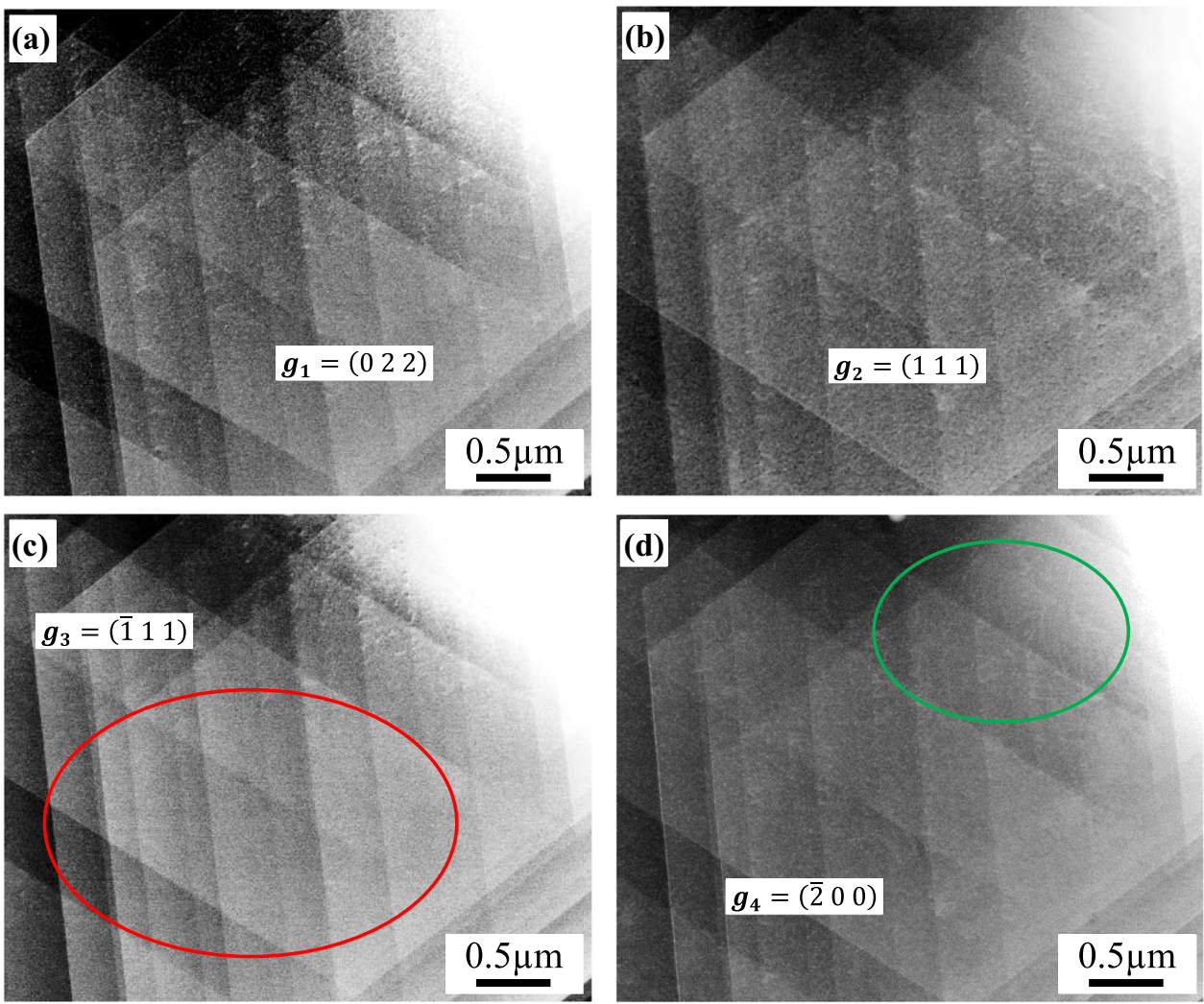

Table 1 Analysis of the Burgers vectors applying the $\mathbf{g} \cdot \mathbf{b}=0$ invisibility criterion (ref. Fig. 5)

\begin{tabular}{cccc}
\hline $\mathbf{g} \cdot \mathbf{b}$ & {$[1 \overline{1} 0]$} & {$[101]$} & {$[011]$} \\
\hline$(022)$ & $\neq 0$ & $\neq 0$ & $\neq 0$ \\
$(111)$ & 0 & $\neq 0$ & $\neq 0$ \\
$(\overline{1} 11)$ & $\neq 0$ & 0 & $\neq 0$ \\
$(\overline{2} 00)$ & $\neq 0$ & $\neq 0$ & 0 \\
\hline
\end{tabular}

symmetry (threefold rotational symmetry and twofold mirror symmetry, which is the same as an equilateral triangle). The symmetry observations on the $\{111\},\{101\}$ and $\{001\}$ grains were confirmed in this study.

\section{Plastic flow depending on the inclination} angle of the slip-plane

The plastic flow direction (Fig. 3) is obtained based on the relation of inclination angles of the slip-planes and pile-up topographies (Fig. 2). The direction of plastic flow is shown with arrows, whose length denotes the magnitude of flow in the two principal directions: forward flow and sideway flow. When the 
slip-plane has an inclination angle to the surface $\alpha \sim 90^{\circ}$, the plane is a barrier to the forward plastic flow and this plane promotes the sideway plastic flow (see (111) and (1 $\left.1_{11}^{-}\right)$slip-planes in $\{101\}$ grain). As shown in Fig. 3, as the inclination angle to the surface decreases (e.g., the three active slip-planes in the $\{111\}$ grain), the sideway flow remains dominant. When the slip-plane has an inclination angle around $55^{\circ}-58^{\circ}$, the barrier effect of the plane on the forward flow is reduced (see $\left(11_{1}^{-}\right)$and (111) slip-planes in $\{001\}$ grain). Once the inclination angle is smaller than $55^{\circ}$, the plasticity will flow mostly forward (see the $\left(11_{1}^{-}\right)$and $\left(1_{1}^{-} 1\right)$ slip-planes in the $\{101\}$ grain). As such, the slip-planes serve as possible 'deflectors' of plasticity transport, i.e., the very inclined planes deflect the plasticity sideways. The three Burgers vectors on each plane are abundant to result in different amounts of dislocation activity for each Burgers vector. The sum of the dislocation activities with different Burgers vectors leads to plastic flow in the most compliant direction. Due to the abundance of Burgers vectors on each plane, the plastic flow will commence in the required direction depending on the plane inclination angle.

A special case of sideway motion occurs if the slipplanes interact and obstruct each other. One example of this obstruction of plastic flow occurs in the $\{101\}$ grain for the slip on the $\left(1_{11}^{-}\right)$and (111) planes (see Fig. $2 b)$. In this case, the motions on the $\left(1_{11}^{-}\right)$and (111) planes are obstructed by the $\left(1_{1}^{-} 1\right)$ and $\left(11_{1}^{-}\right)$ planes, respectively. These interactions result in more plastic flow away from the interaction point. As a result, the pile-ups become non-symmetric: compliant plasticity spreading on one side and constrain on the other side. A more detailed look in slip-plane interaction is given in "Dislocation motion and interaction" section.

The experiments show that the transition inclination angle between forward and sideway dislocation motion is around $55^{\circ}-58^{\circ}$ irrespective of the crystal rotation angle. Moreover, we discussed how the abundance of Burger's vectors allows realizing the plastic flow in the most compliant direction. We carried out phenomenological FEM simulations to further investigate the inclination angle dependence on the plastic flow. These simulations use the simplest possible model to account for the pile-up shape and neglect the crystal orientation. A series of CP-
FEM simulations of different orientations is required to confirm this experimental finding. However, such study is very time-consuming. By comparing the experimental and numerical findings, we determine whether the simple phenomenological model is sufficient in explaining the transition inclination angle.

We used the 2D plane-strain formulation in Abaqus Dynamic Explicit and the large strain formulation that takes the pile-up formation into account. The indenter was modeled as an infinitely hard surface that interacted with the surface. The indentersurface interaction prevented indenter penetration, and a minor friction coefficient (0.0002) prevented numerical instabilities. The material was modeled as an elastic-plastic metal with a Young's modulus of $210 \mathrm{GPa}$, density of $7.85 \mathrm{~g} / \mathrm{cm}^{3}$ and a Poisson ratio of 0.3 . The flow stress is $600 \mathrm{MPa}$, and the linear hardening leads to a flow stress of $680 \mathrm{MPa}$ at $10 \%$ plastic strain. A time step of $10 \mathrm{~ms}$ ensured that the kinetic energy is minimal compared to the total energy; thus, the elastic waves do not significantly influence the results. The stress components from the left and right side of the indentation axis were averaged to further reduce the influence of the elastic waves. Therefore, we did not use the symmetric boundary conditions in the indentation axis. The material consisted of 11250 nodes to achieve smooth stress contours. The maximal shear stress is shown in Fig. 6a.

The stress state in the indentation centerline is governed by the symmetry of boundary conditions. On the sample surface, the stress state is dominated by the free-surface boundary conditions. Hence, material points on the imprint shoulder are of most interest as the stress state at these points determines the plastic flow. Toward this goal, three FEM nodes on the imprint shoulder were chosen and the resolved shear stresses were calculated at increments of $1^{\circ}$ between $0^{\circ}$ and $90^{\circ}$. Due to conservation of angular momentum, identical stresses occur on planes with $90^{\circ}$ separation. For one FEM node, the vectors in Fig. 6a show the resolved shear stress and the identical stresses every $90^{\circ}$. The magnitude of the resolved shear stress is shown in Fig. $6 \mathrm{~b}$ for the three points as a function of the inclination angle to the horizontal. The shear stresses change sign at an inclination angle of roughly $60^{\circ}$ for all three points.

If the inclination angle is small, it is favorable for the plasticity to shear away from the imprint. Positive shear stress is in the positive $x$-direction on the plane with the positive $y$-direction, see Fig. 6a. The driving 


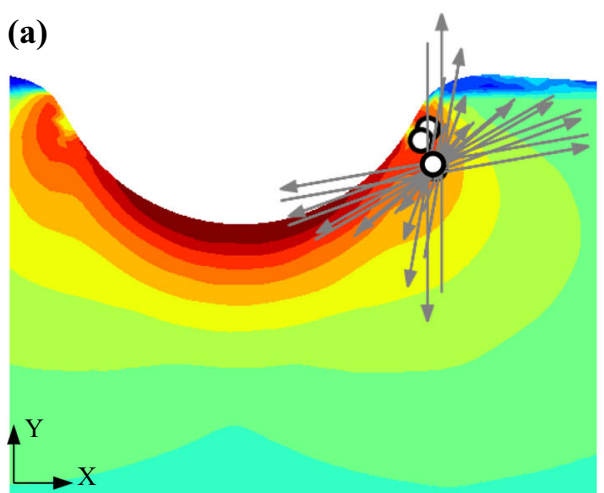

Figure 6 a Maximum shear stress distribution during indentation according to elastic-plastic FEM simulations. Three points on the imprint shoulder are used for evaluation of the resolved shear stress. The arrow lengths on the lowest of the points denote the

force for dislocation forward motion, i.e., positive resolved shear stress, decreases but remains positive with increasing inclination angle, see Fig. $6 \mathrm{~b}$. Therefore, forward plasticity motion dominates in this inclination angle regime. This observation agrees to the experimental finding that the forward motion occurs for small inclination angles (below $55^{\circ}$ ). The simulation predicts that forward motion becomes impossible for inclination angles above $60^{\circ}$, which supports the experimental observation, which showed no forward motion above $58^{\circ}$.

It should be noted that other FEM nodes have different stress states and different transition angles. The precise evaluation of the transition angle is of no benefit for this study because the experimentally obtained angles have an uncertainty of around $1^{\circ}$ due to the EBSD calibration. The phenomenological model predicts the transition angle with an accuracy of $5^{\circ}$ and highlights that the inversion of the resolved shear stress is the cause for the transition between forward and sideway motion. Therefore, this simple phenomenological FEM simulation is reliable in explaining the experimental finding and is more time efficient than CP-FEM.

\section{Dislocation motion and interaction}

The interaction of dislocations depends on the local stress state and the involved shear planes. Hence, the dislocation interaction is different for each site around the imprint perimeter and is different for each grain orientation. Here, we discuss one case of dislocation glide plane interaction to highlight the

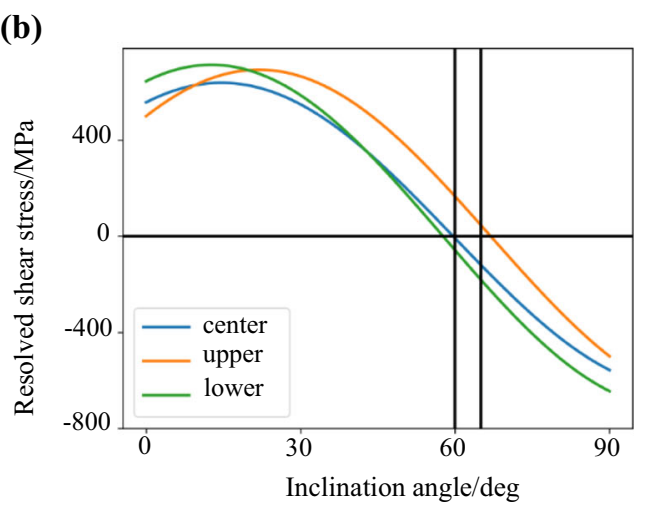

magnitude of the resolved shear stress in the particular direction. b Resolved shear stress for the three points depending on the inclination angle of the direction.

possibilities of experimental methods and the corresponding analysis, and the requirement for more involved numerical simulations to account for the precise stress state. Toward this goal, we focus on the $\{101\}$ grain and on the lower left-side corner of the imprint, see Fig. 4.

After indentation with $2 \mathrm{mN}$, the slip-step pattern is dominated by multiple parallelograms (i.e., slip on (111) and $\left(1_{11}^{-}\right)$planes). Slip on the positively inclined $\left(11_{1}^{-}\right)$and $\left(1_{1}^{-} 1\right)$ planes is constrained by the outside parallelogram (Fig. 7a, c). In the next deformation state, the slip on the most distant (111) slip-plane-denoted as ' $\mathrm{I}$ '-from the imprint center breaks the obstruction by the $\left(1_{1}^{-} \overline{1}\right)$ planes at the bottom left corner. Thereafter, this (111) slip-plane grows outside of the parallelogram, as sketched in Fig. 7d. The distance of slip-step ' $\mathrm{I}$ ' is $\sim 700 \mathrm{~nm}$ to the (111) centerline. As the indenter penetrates further, more dislocations multiplicate and move on the unobstructed (111) slip-plane ' $\mathrm{I}$ ', resulting in the highest pile-up forming along step ' $\mathrm{I}$ ' close to the imprint. Additionally, more (111) slips are activated at the larger distances from the centerline. The corresponding steps ' $\mathrm{II}$ ' have lower pile-up heights than the pile-up at step ' $\mathrm{I}$ '.

After indentation with $80 \mathrm{mN}$, the slip-steps on the bottom (111) slip-planes are longer than on the upper planes, which reveal less obstructed dislocation motion on the bottom planes, as shown in Fig. $4 \mathrm{~b}$. The Burgers vector $\left[0_{11}^{--}\right]\left(11_{1}^{-}\right)$of the known dislocations in the green ellipse area, see "Interaction 
Figure 7 a SEM image of the $2 \mathrm{mN}$ imprints, $\mathbf{b}$ the corresponding Thompson tetrahedron notation and $\mathbf{c}-\mathbf{e}$ schematic diagram of slip-step evolution in the bottom-left pile-up of grain $\{101\}$ : c no (111) slip-step, $\mathbf{d}$ only one (111) slip-step and e more than one (111) slip-step grow outside of the multiparallelogram: the corresponding SEM images of $(\mathbf{a}, \mathbf{b})$ are shown in Fig. 4 .

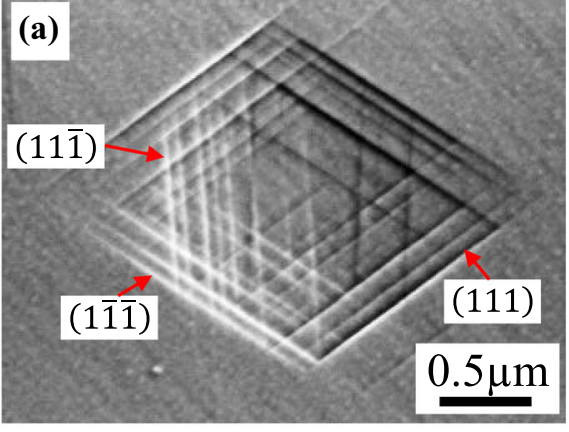

(b)

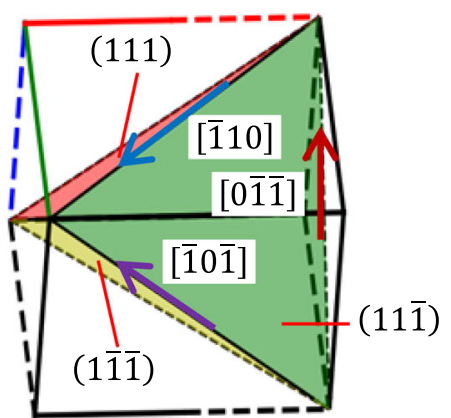

(c)

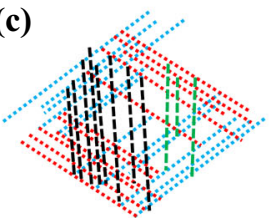

(d)

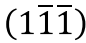

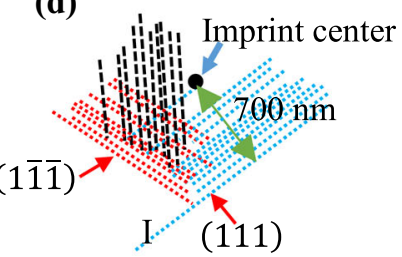

(e)

$(11 \overline{1})$

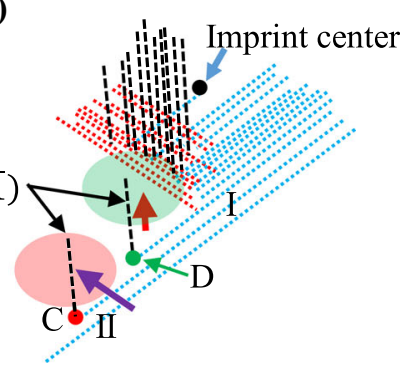

between slip-planes" section, implies that the plasticity flows on the $\left(11_{1}^{-}\right)$slip-planes along the $\left[0_{11}^{--}\right]$ direction to the green area (see Fig. 7e). Moreover, the plasticity will flow on the $\left(11_{1}^{-}\right)$slip-plane along the $\left[{ }_{1}^{-} 0_{1}^{-}\right]$direction to the red area, as sketched in Fig. 7e. Three Burgers vectors exist on the bottom (111) slipplanes, only one of which, i.e., $[-10]$, leads to the plasticity flowing forward (see Fig. $7 \mathrm{~b}$ ). Hence, the dislocation motion on the $(111)$ and $\left(11_{1}^{-}\right)$slip-planes leads to glissile junctions at the interactions ' $\mathrm{C}$ ' and 'D,' as shown in Figs. 4 and 7. Further CP-FEM or DDD simulations are required to investigate the stress field changes and the dislocation motion in these two areas.

\section{Slip-step and pile-up evaluation}

The schematic diagram of slip-step pattern evolutions in the $\{111\},\{101\}$ and $\{001\}$ grains is shown in Fig. 8. In grain $\{111\}$, the (111) slip-plane is almost parallel to the sample surface and does not exhibit slip-steps as the Schmidt factor is neglectable. At the beginning of indentation, the dislocations travel on the positively inclined $\left(1_{1}^{-} 1\right),\left(11_{1}^{-}\right)$and $\left({ }_{11}^{-} 1\right)$ slipplanes. Because the inclination angles $\left(\alpha=\sim 70^{\circ}\right)$ of these planes are larger than the transition angle $\left(55^{\circ}-58^{\circ}\right)$, the plastic forward flow is obstructed, resulting in the sideway flow. The accompanying mutual interactions from these planes lead to the pattern of stacked triangles (Fig. 8a). As the load increases, the pile-ups build up at the triangle vertices and slip commences on negatively inclined slipplanes (Fig. 8b). Afterward, the slip-step pattern increases by geometric similarity (Fig. 8c).

As the indenter presses into grain $\{101\}$, all four slip-planes are activated (Fig. 8d). The (111) and $\left(11_{11}^{-}\right)$slip-planes are almost perpendicular to the surface, and the resolved shear stresses on these planes are much higher than on another two slipplanes, which results in preferential dislocation motion on these planes. The interaction of dislocations from these two planes results in the slip-step parallelogram formation. The dislocations also travel on positively inclined $\left(11_{1}^{-}\right)$and $\left(1_{1}^{-} 1\right)$ planes. As the load increases, one sideway flow on the (111) and $\left(1_{11}^{-}\right)$planes is constrained by the $\left(11_{1}^{-}\right)$and $\left(1_{1}^{-} 1\right)$ planes and the plasticity is transported to the other direction on these two planes. Together with the forward flow on the $\left(11_{1}^{-}\right)$and $\left(1_{1}^{-} 1\right)$ planes $\left(\alpha \sim 35^{\circ}\right)$, the plastic flow results in the formation of four pile-ups (Fig. 2). Please note that the slip on the $\left(11_{1}^{-}\right)$and $\left(1_{1}^{-} 1\right)$ planes is also obstructed by the (111) and $\left(1_{11}^{-}\right)$planes. Finally, a butterfly pattern forms and grows (Fig. 8e, f). 
Figure 8 Schematic diagram of the slip-step evolution during the indentations into grains $\{111\},\{101\}$ and $\{001\}$ [the corresponding SEM images are given as supplemental information].

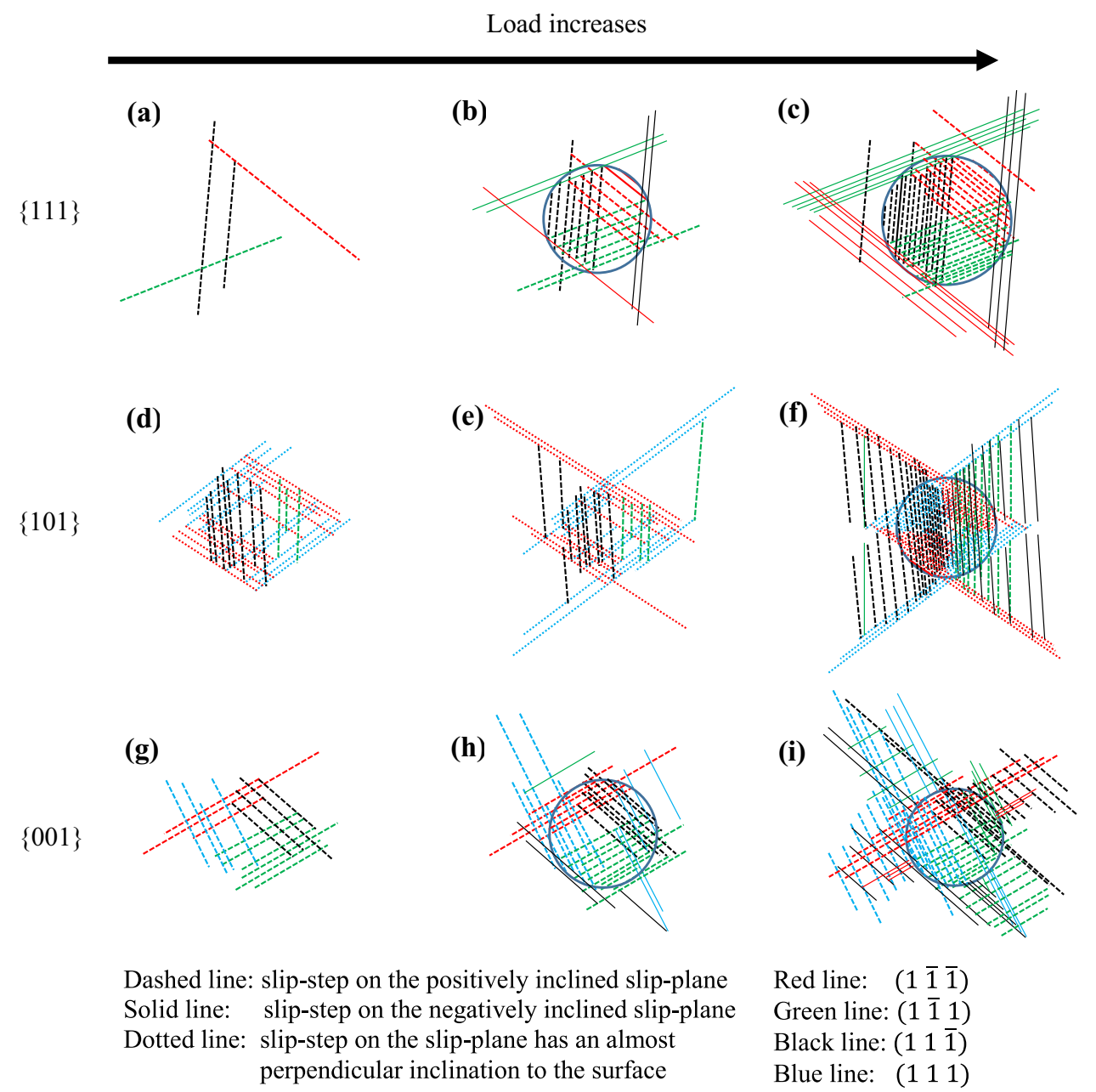

During the indentation into the $\{001\}$ grain, slip commences first on the four positively inclined slipplanes (Fig. 8g) and then on the negatively inclined $\left(1_{1}^{-} 1\right)$ and $(111)$ and $\left(11_{1}^{-}\right)$(Fig. $8 \mathrm{~h}$ ). The sideway motion of plasticity on the positively inclined (111) and $\left(11_{1}^{-}\right)$slip-planes $\left(58^{\circ}\right.$ and $\left.55^{\circ}\right)$ results in the formation of pile-up I (Fig. 2f). The plasticity on these two slip-planes is additionally transported along the forward direction (Fig. 3) and contributes to the growth of the pile-ups III and IV, respectively. The plasticity flows along the two sideway directions on the $\left(1_{11}^{-}\right)$planes $\left(73^{\circ}\right)$ and enables the further expansion of the pile-ups III and IV away from the imprint (Fig. 8h). Finally, the cross-shaped slip-steps are generated and grow (Fig. 8i).

\section{Conclusions}

This study aimed at thoroughly understanding the deformation mechanisms during nanoindentation, which will be used to characterize single phases of materials in the future. Moreover, the study aimed at learning the deformation caused by a hard precipitate on a ductile counter surface during contact and tribological loading. The dependence of the slip-plane inclination angle on plastic flow during nanoindentation was studied by analyzing the slip-step and pile-up evolution for three grains of the austenitic stainless steel. The following conclusions are drawn:

1. The pile-up formation can be understood by the plastic flow on slip-planes.

2. Plastic flow is mainly determined by the inclination angle of the slip-plane with respect to the surface. The flow direction transforms from the forward motion to the sideway at a transition angle of $55^{\circ}-58^{\circ}$. 
3. Large strain FEM simulations verified an inversion of the resolved shear stress at this transition angle.

4. The shape of the pile-up is also determined by the interaction of the glide planes. The formation of dislocation junctions and locks constrains the spread of plasticity and the size of the pile-up.

\section{Acknowledgements}

Open access funding provided by Projekt DEAL. The authors appreciate the funding of the Deutsche Forschungsgemeinschaft DFG within Project BR3947/5-1. The authors thank Dr. Dayong An for the measurement of and discussion on Burgers vectors using ECCI.

Electronic supplementary material: The online version of this article (https://doi.org/10.1007/s108 53-020-04646-y) contains supplementary material, which is available to authorized users.

Open Access This article is licensed under a Creative Commons Attribution 4.0 International License, which permits use, sharing, adaptation, distribution and reproduction in any medium or format, as long as you give appropriate credit to the original author(s) and the source, provide a link to the Creative Commons licence, and indicate if changes were made. The images or other third party material in this article are included in the article's Creative Commons licence, unless indicated otherwise in a credit line to the material. If material is not included in the article's Creative Commons licence and your intended use is not permitted by statutory regulation or exceeds the permitted use, you will need to obtain permission directly from the copyright holder. To view a copy of this licence, visit http://creativecommons.org/licen ses/by/4.0/.

\section{References}

[1] Schuh CA (2006) Nanoindentation studies of materials. Mater Today 9(5):32-40

[2] Fischer-Cripps AC (2006) Critical review of analysis and interpretation of nanoindentation test data. Surf Coat Technol 200(14-15):4153-4165
[3] Fischer-Cripps AC (2011) Factors affecting nanoindentation test data. In: Nanoindentation. Mechanical engineering series. Springer, New York, NY, pp 77-104

[4] Oliver WC, Pharr GM (1992) An improved technique for determining hardness and elastic-modulus using load and displacement sensing indentation experiments. J Mater Res 7(6):1564-1583

[5] Oliver WC, Pharr GM (2004) Measurement of hardness and elastic modulus by instrumented indentation: advances in understanding and refinements to methodology. J Mater Res 19(1):3-20

[6] Nix WD, Gao HJ (1998) Indentation size effects in crystalline materials: a law for strain gradient plasticity. J Mech Phys Solids 46(3):411-425

[7] Gao H, Huang Y, Nix WD, Hutchinson JW (1999) Mechanism-based strain gradient plasticity-I. Theory. J Mech Phys Solids 47(6):1239-1263

[8] Maier-Kiener V, Schuh B, George EP et al (2017) Insights into the deformation behavior of the $\mathrm{CrMnFeCoNi}$ highentropy alloy revealed by elevated temperature nanoindentation. J Mater Res 32(14):2658-2667

[9] Chen TY, Tan LZ, Lu ZZ et al (2017) The effect of grain orientation on nanoindentation behavior of model austenitic alloy Fe-20Cr-25Ni. Acta Mater 138:83-91

[10] Song H, Yavas H, Van der Giessen E et al (2019) Discrete dislocation dynamics simulations of nanoindentation with pre-stress: hardness and statistics of abrupt plastic events. J Mech Phys Solids 123:332-347

[11] Ganji RS, Karthik PS, Rao KBS et al (2017) Rajulapati, Strengthening mechanisms in equiatomic ultrafine grained AlCoCrCuFeNi high-entropy alloy studied by micro- and nanoindentation methods. Acta Mater 125:58-68

[12] Rester M, Motz C, Pippan R (2008) Indentation across size scales - a survey of indentation-induced plastic zones in copper 111 single crystals. Scr Mater 59(7):742-745

[13] Jakob S, Leitner A, Lorich A et al (2019) (2019) Influence of crystal orientation and Berkovich tip rotation on the mechanical characterization of grain boundaries in molybdenum. Mater Des 182:107998

[14] Chen SW, Miyahara Y, Nomoto A (2018) Crystallographic orientation dependence of nanoindentation hardness in austenitic phase of stainless steel. Philos Mag Lett 98(11):473-485

[15] Higashino S, Miyake M, Takahashi A et al (2017) Evaluation of the hardness and Young's modulus of electrodeposited Al-W alloy films by nano-indentation. Surf Coat Technol 325:346-351

[16] Huang Y, Gao H, Nix WD (2000) Mechanism-based strain gradient plasticity-II. Analysis. J Mech Phys Solids 48(1):99-128 
[17] Tromas C, Gaillard Y, Woirgard J (2006) Nucleation of dislocations during nanoindentation in $\mathrm{MgO}$. Philos Mag 86(33-35):5595-5606

[18] Velednitskaya M, Rozhanskii V, Comolova L et al (1975) Investigation of the deformation mechanism of $\mathrm{MgO}$ crystals affected by concentrated load. Phys Status Solidi (a) 32(1):123-132

[19] Tromas C, Girard J, Audurier V (1999) Study of the low stress plasticity in single-crystal $\mathrm{MgO}$ by nanoindentation and atomic force microscopy. $\mathrm{J}$ Mater Sci 34(21):5337-5342. https://doi.org/10.1023/A: 1004705206482

[20] Nibur KA, Bahr DF (2003) Identifying slip systems around indentations in FCC metals. Scr Mater 49(11):1055-1060

[21] Nibur KA, Akasheh F, Bahr DF (2007) Analysis of dislocation mechanisms around indentations through slip step observations. J Mater Sci 42(3):889-900. https://doi.org/10. 1007/s10853-006-0007-x

[22] Bolshakov A, Pharr GM (1998) Influences of pileup on the measurement of mechanical properties by load and depth sensing indentation techniques. $J$ Mater Res 13(4):1049-1058

[23] McElhaney KW, Vlassak JJ, Nix WD (1998) Determination of indenter tip geometry and indentation contact area for depth-sensing indentation experiments. J Mater Res 13(5):1300-1306

[24] Giannakopoulos AE, Suresh S (1999) Determination of elastoplastic properties by instrumented sharp indentation. Scr Mater 40(10):1191-1198

[25] Taljat B, Pharr GM (2004) Development of pile-up during spherical indentation of elastic-plastic solids. Int J Solids Struct 41(14):3891-3904

[26] Eidel B (2011) Crystal plasticity finite-element analysis versus experimental results of pyramidal indentation into (001) fcc single crystal. Acta Mater 59(4):1761-1771

[27] Casals O, Ocenasek J, Alcala J (2007) Crystal plasticity finite element simulations of pyramidal indentation in copper single crystals. Acta Mater 55(1):55-68

[28] Wang Y, Raabe D, Kluber C et al (2004) Orientation dependence of nanoindentation pile-up patterns and of nanoindentation microtextures in copper single crystals. Acta Mater 52(8):2229-2238

[29] Zaafarani N, Raabe D, Singh RN (2006) Three-dimensional investigation of the texture and microstructure below a nanoindent in a $\mathrm{Cu}$ single crystal using 3D EBSD and crystal plasticity finite element simulations. Acta Mater 54(7):1863-1876

[30] Demir E, Raabe D, Zaafarani N (2009) Investigation of the indentation size effect through the measurement of the geometrically necessary dislocations beneath small indents of different depths using EBSD tomography. Acta Mater 57(2):559-569

[31] Fivel MC, Robertson CF, Canova GR et al (1998) Threedimensional modeling of indent-induced plastic zone at a mesoscale. Acta Mater 46(17):6183-6194

[32] Robertson CF, Fivel MC (1999) A study of the submicron indent-induced plastic deformation. $\mathrm{J}$ Mater Res 14(6):2251-2258

[33] Gagel J, Weygand D, Gumbsch P (2016) Formation of extended prismatic dislocation structures under indentation. Acta Mater 111:399-406

[34] Chang HJ, Fivel M, Rodney D et al (2010) Multiscale modelling of indentation in FCC metals: from atomic to continuum. Cr Phys 11(3-4):285-292

[35] Begau C, Hua J, Hartmaier A (2012) A novel approach to study dislocation density tensors and lattice rotation patterns in atomistic simulations. J Mech Phys Solids 60(4):711-722

[36] Begau C, Hartmaier A, George EP et al (2011) Atomistic processes of dislocation generation and plastic deformation during nanoindentation. Acta Mater 59(3):934-942

[37] Stukowski A, Arsenlis A (2012) On the elastic-plastic decomposition of crystal deformation at the atomic scale. Model Simul Mater Sci 20(3):035012

[38] Varillas J, Ocenasek J, Torner J et al (2017) Unraveling deformation mechanisms around FCC and BCC nanocontacts through slip trace and pileup topography analyses. Acta Mater 125:431-441

[39] Lu CY, Yang TN, Jin K et al (2018) Enhanced void swelling in $\mathrm{NiCoFeCrPd}$ high-entropy alloy by indentation-induced dislocations. Mater Res Lett 6(10):584-591

[40] Cackett AJ, Hardie CD, Lim JJH et al (2019) Spherical indentation of copper: crystal plasticity vs experiment. Materialia 7:100368

[41] Xia W, Dehm G, Brinckmann S (2019) Unraveling indentation-induced slip steps in austenitic stainless steel. Mater Des 183:108169

[42] Schramm RE, Reed RP (1975) Stacking fault energies of seven commercial austenitic stainless steels. Metall Trans A 6(7): 1345

[43] Zaefferer S, Elhami NN (2014) Theory and application of electron channelling contrast imaging under controlled diffraction conditions. Acta Mater 75:20-50

[44] Liu Y, Varghese S, Ma J et al (2008) Orientation effects in nanoindentation of single crystal copper. Int $\mathrm{J}$ Plast 24(11):1990-2015

[45] Ju SP, Wang CT, Chien CH et al (2007) The nanoindentation responses of nickel surfaces with different crystal orientations. Mol Simul 33(11):905-917

[46] Renner E, Gaillard Y, Richard F et al (2016) Sensitivity of the residual topography to single crystal plasticity parameters 
in Berkovich nanoindentation on FCC nickel. Int $\mathrm{J}$ Plast 77:118-140

[47] Ha S, Jang JH, Kim K (2017) Finite element implementation of dislocation-density-based crystal plasticity model and its application to pure aluminum crystalline materials. Int $\mathrm{J}$ Mech Sci 120:249-262
Publisher's Note Springer Nature remains neutral with regard to jurisdictional claims in published maps and institutional affiliations. 